\title{
Silencing of FGF-21 expression promotes hepatic gluconeogenesis and glycogenolysis by regulation of the STAT3-SOCS3 signal
}

\author{
Cong Wang ${ }^{1}$, Jihuan Dai ${ }^{1}$, Mengliu Yang ${ }^{1}$, Guangjiang Deng ${ }^{2}$, Shengnan $\mathrm{Xu}^{1}$, Yanjun Jia ${ }^{1}$, \\ Guenther Boden ${ }^{3}$, Zhongmin A. Ma ${ }^{4}$, Gangyi Yang ${ }^{2}$ and Ling $\mathrm{Li}^{1}$ \\ 1 Key Laboratory of Diagnostic Medicine (Ministry of Education) and Department of Clinical Biochemistry, College of Laboratory Medicine, \\ Chongqing Medical University, 400016, China \\ 2 Department of Endocrinology, the Second Affiliated Hospital, Chongqing Medical University, 400010, China \\ 3 Division of Endocrinology/Diabetes/Metabolism and Clinical Research Center, Temple University School of Medicine, Philadelphia, PA \\ 19140, USA \\ 4 Technology Transfer Center, University of Michigan, Ann Arbor, MI, USA
}

\section{Keywords \\ FGF-21 knockdown; insulin resistance; liver glucose fluxes; SOCS3; STAT3 \\ Correspondence \\ L. Li, Department of Clinical Biochemistry, College of Laboratory Medicine, Chongqing Medical University, 400010 Chongqing, \\ China \\ Fax: +862368485005 \\ Tel: +86 2368485216 \\ E-mail: lingli31@hotmail.com}

(Received 30 September 2013, revised 29 January 2014, accepted 25 February 2014)

doi:10.1111/febs.12767
Insulin resistance is a metabolic disorder associated with type 2 diabetes. Recent reports have shown that fibroblast growth factor-21 (FGF-21) plays an important role in the progression of insulin resistance. However, the biochemical and molecular mechanisms by which changes in FGF-21 activation result in changes in the rates of hepatic gluconeogenesis and glycogenolysis remain to be elucidated. In this study, we developed adenovirus-mediated shRNA against FGF-21 to inhibit FGF-21 expression in ApoE knockout mice. Using this mouse model, we determined the effects of FGF-21 knockdown in vivo on hepatic glucose production, gluconeogenesis and glycogenolysis, and their relationship with the signal transducer and activator of transcription 3 (STAT3)/suppressor of cytokine signaling 3 (SOCS3) signal pathways. We show that liver-specific knockdown of FGF-21 in high-fat diet-fed ApoE knockout mice resulted in a $39 \%$ increase in glycogenolysis and a $75 \%$ increase in gluconeogenesis, accompanied by increased hepatic expression of glucose-6-phosphatase and phosphoenolpyruvate carboxykinase. Furthermore, FGF-21 knockdown decreased phosphorylation of STAT3 and SOCS3 expression in high-fat diet-fed mice. Our data suggest that hepatic FGF-21 knockdown increases gluconeogenesis and glycogenolysis by activation of glucose-6phosphatase and phosphoenolpyruvate carboxykinase via the STAT3/ SOCS3 pathway, ultimately leading to exacerbation of hepatic insulin resistance.

\section{Introduction}

Fibroblast growth factor-21 (FGF-21) is a recently identified member of the FGF family, and is preferentially expressed in the liver [1]. We previously demonstrated that plasma FGF-21 levels are elevated in patients with type 2 diabetes and patients with diabetic ketoacidosis [1,2], and decreased in response to rosiglitazone treatment [3]. In fact, in both dietinduced obese mice [4] and genetically obese $o b / o b$ mice, FGF-21 plasma levels and expression in white adipose tissue and liver are increased [5]. It is therefore believed that FGF-21 resistance occurs in obesity and diabetes [6]. In obese and diabetic rodents, treatment with pharmacological doses of FGF-21 reduced body fat and improved glucose tolerance, insulin sensitivity and lipid levels [7-11]. Nevertheless, the physiological roles of FGF- 21 in the 
development of insulin resistance remain to be established. In the present study, we have developed an adenovirus-mediated RNAi technique in which shRNA against FGF-21 were used to inhibit FGF-21 expression in vivo. The major advantages of shRNA silencing of gene expression in a normal adult animal are its efficiency and specificity, avoiding the confounding compensatory developmental effects often associated with gene knockout mouse models.

It has been recognized that lipid disorders are the earliest events in development of insulin resistance and type 2 diabetes [12]. ApoE knockout (KO) mice develop severe atherosclerosis and hypercholesterolemia on a standard chow diet (SCD), and are widely used as a model of insulin resistance when fed with high-fat diet (HFD) [13]. HFD-fed ApoE KO mice are also a useful model for studying the lipid disorders and insulin resistance associated with increased tissue expression and plasma levels of FGF-21 [14,15]. Type 2 diabetes and insulin resis-tance are believed to arise from a combination of genetic and environmental factors. Therefore, this mouse model, in which insulin resistance is developed by feeding an HFD (environmental factor) to ApoE KO mice (genetic factor), closely simulates human insulin resistance conditions, and was used to determine the effect of elevation of circulating FGF-21 on the onset of insulin resistance.

Recent studies have shown that the signal transducer and activator of transcription 3 (STAT3)/suppressor of cytokine signaling 3 (SOCS3) pathway regulates signal transduction of many factors related to insulin sensitivity and glucose metabolism [16,17]. As FGF-21 is a metabolic regulator, it is important to determine whether the STAT3/SOCS3 pathway is involved in the metabolic effects of FGF-21. In this study, we examined the effect of FGF-21 knockdown on the relative contribution of gluconeogenesis and glycogenolysis to the glucose-6-phosphatase (G6Pase) flux and the STAT3/SOCS3 pathway using adenovirus-mediated shRNA of FGF-21 in HFD-fed mice.

\section{Results}

\section{RNAi-mediated FGF-21 knockdown decreased hepatic expression and plasma levels of FGF-21}

A previously validated shRNA sequence against FGF-21[18] was introduced into an adenovirus vector (Ad-shFGF-21), and its relatively silencing efficiency was compared with that of a control sequence (AdshGFP). Treatment with Ad-shFGF-21 achieved 43\% and $50 \%$ reductions in the levels of FGF- 21 mRNA and protein in the liver $(P<0.01$, Fig. $1 \mathrm{~A}, \mathrm{C})$ but no reduc- tion was observed in muscle (Fig. S1). When compared with HFD-fed ApoE KO mice (HF group) and HFDfed plus Ad-shGFP-treated ApoE KO mice (GFP group), HFD-fed plus Ad-shFGF-21 treated mice (HFG group) showed a $30 \%$ reduction in the plasma FGF-21 levels $(P<0.01$, Fig. 1B), demonstrating that AdshFGF-21 effectively reduces FGF-21 expression in vivo.

\section{Effect of RNAi-mediated FGF-21 knockdown on metabolic parameters}

We next assessed the effects of FGF-21 knockdown on metabolic parameters. Values for body weight and many plasma parameters, including fasting blood glucose, lipids, insulin and hepatic triglycerides (TG), were significantly higher in ApoE KO mice fed the HFD than in their littermates fed a standard chow diet (SCD) (NF group) $(P<0.05$ or $P<0.01$, Table 1$)$. There were no significant differences in fasting blood glucose, total cholesterol (TC), low-density lipoprotein cholesterol (LDL-C), high-density lipoprotein cholesterol (HDL-C), free fatty acids (FFA), or insulin and hepatic TG between the HF and GFP groups. However, the plasma levels of fasting blood glucose, TC, LDL-C, FFA, insulin and hepatic TG in the HFG group were significantly higher than in the $\mathrm{HF}$ and GFP groups $(P<0.05$ or $P<0.01$, Table 1$)$; the HDL-C level was significantly lower $(P<0.05)$.

\section{Effect of RNAi-mediated FGF-21 knockdown on glucose homeostasis and insulin sensitivity}

To assess the effects of FGF-21 knockdown on glucose homeostasis and insulin action in HFD-induced insulin resistance, we performed euglycemic/hyperinsulinemic clamp studies in the four experimental groups (Fig. 2A, B). Plasma glucose was clamped at $\sim 6 \mathrm{~mm}$ in all groups (Fig. S2A), whereas plasma insulin levels were increased approximately four- to sixfold compared with basal values. Despite identical insulin infusion rates $\left(5 \mathrm{mU} \cdot \mathrm{kg}^{-1} \cdot \mathrm{min}^{-1}\right)$, plasma insulin levels during the clamps were higher in the HFG group than in the other groups $(P<0.01$, Fig. $2 \mathrm{C})$. TG, TC and FFA levels were suppressed by hyperinsulinemia in all animals $(P<0.01)$, but remained significantly higher in the HFG group $(P<0.05$ or $P<0.01$, Fig. $2 \mathrm{D}-\mathrm{F})$. Figure $3 \mathrm{~A}$ shows the glucose infusion rates required to maintain euglycemia in the various groups. The glucose infusion rate was decreased by $\sim 76 \%$ in mice fed the HFD (HF group) compared with mice fed the SCD (NF group, $P<0.01)$. However, the glucose infusion rate (GIR) was significantly decreased in the HFG group compared with the GFP group $(P<0.01)$, indicating 
A

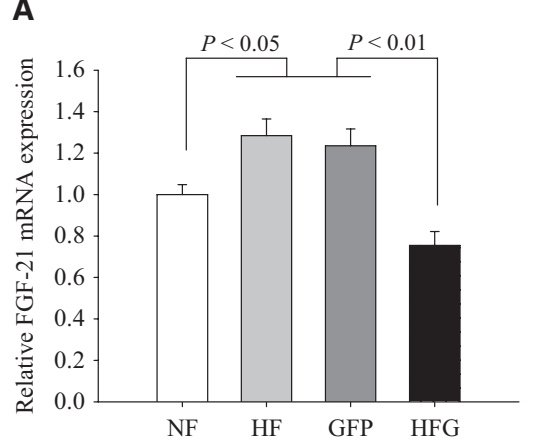

B



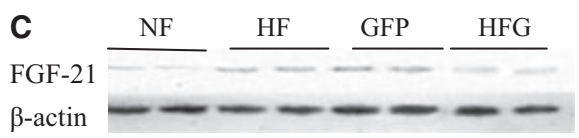

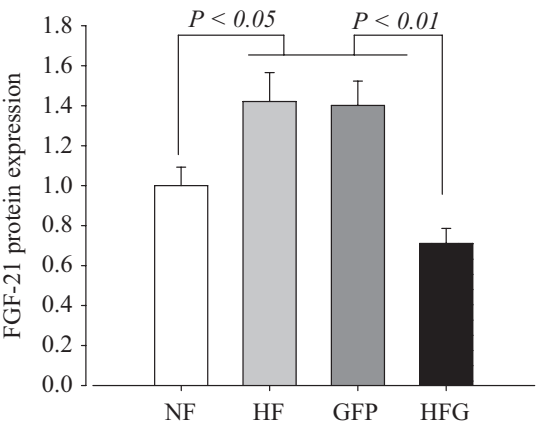

Fig. 1. Adenovirus-mediated FGF-21 knockdown in HFD-fed ApoE KO mice. Mice were injected with Ad-shFGF-21 or Ad-sh GFP $\left(1 \times 10^{9}\right.$ plaque-forming units $\mathrm{g}^{-1}$ body weight) as described in the text. (A) Relative FGF-21 mRNA levels in the liver. (B) Plasma FGF-21 levels. (C) FGF-21 protein level in the liver. All data are the results of at least five independent experiments. NF, SCD-fed mice; HF, HFDfed ApoE KO mice; GFP, HFD-fed ApoE KO mice treated with Ad-sh GFP; HFG, HFD-fed ApoE KO mice treated with AdshFGF-21. Values are means $\pm S E$, and are expressed as fold changes relative to the levels in the NF group.

Table 1. Characteristics of the experimental groups.

\begin{tabular}{|c|c|c|c|c|}
\hline & NF & $\mathrm{HF}$ & GFP & HFG \\
\hline Body weight (g) & $27.4 \pm 1.2$ & $29.4 \pm 1.3^{* *}$ & $28.5 \pm 1.2^{* *}$ & $28.7 \pm 1.5^{* *}$ \\
\hline $\mathrm{FBG}(\mathrm{mm})$ & $5.9 \pm 0.8$ & $7.7 \pm 0.8^{* *}$ & $7.7 \pm 0.4^{* *}$ & $8.4 \pm 0.5^{* *, \uparrow, \S}$ \\
\hline $\begin{array}{l}\text { Fasting insulin } \\
\left(\mathrm{mU} \cdot \mathrm{L}^{-1}\right)\end{array}$ & $82.3 \pm 6.3$ & $111.2 \pm 13.0^{* *}$ & $112.1 \pm 13.6^{* *}$ & $133.1 \pm 10.4^{* *+, s \S}$ \\
\hline $\mathrm{TC}(\mathrm{mm})$ & $12.2 \pm 3.1$ & $21.9 \pm 1.8^{* *}$ & $20.9 \pm 2.4^{* *}$ & $24.8 \pm 2.06^{* *, \dagger, \S}$ \\
\hline $\mathrm{TG}(\mathrm{mm})$ & $1.2 \pm 0.2$ & $2.3 \pm 0.2^{* *}$ & $2.2 \pm 0.2^{* *}$ & $2.6 \pm 0.2^{* *+\ldots \S}$ \\
\hline FFA (mм) & $2.21 \pm 0.34$ & $3.1 \pm 0.09^{* *}$ & $3.10 \pm 0.10 * *$ & $3.5 \pm 0.10^{* *}, \$ \S \S$ \\
\hline HDL-C (mм) & $2.5 \pm 0.5$ & $4.6 \pm 0.8^{* *}$ & $4.5 \pm 0.5^{* *}$ & $3.9 \pm 0.3^{* * \hbar . \$ \S}$ \\
\hline LDL-C (mм) & $6.0 \pm 1.7$ & $14.0 \pm 2.1^{* *}$ & $13.7 \pm 2.2^{* *}$ & $16.6 \pm 1.5^{* *+1,8 \S}$ \\
\hline $\begin{array}{l}\text { Hepatic TG } \\
\left.\text { (mg } \cdot \mathrm{g}^{-1} \text { liver }\right)\end{array}$ & $14.5 \pm 2.3$ & $39.2 \pm 3.04^{* *}$ & $41.0 \pm 3.27^{* *}$ & $46.1 \pm 3.71^{* *}, \% \S$ \\
\hline $\begin{array}{l}\text { Hepatic glycogen } \\
\text { (mg } \cdot \mathrm{g}^{-1} \text { liver) }\end{array}$ & $22.4 \pm 3.13$ & $16.1 \pm 1.92^{* *}$ & $15.9 \pm 1.37^{* *}$ & $13.8 \pm 1.1^{* *, \dagger}, \S$ \\
\hline
\end{tabular}

FBG, fasting blood glucose; TC, total cholesterol; TG, triglycerides; FFA, free fatty acids; HDL-C, high density lipoprotein cholesterol; LDL-C, low density lipoprotein cholesterol; NF, SCD-fed mice; HF, HFD-fed ApoE KO mice; GFP, HFD-fed ApoE KO mice treated with Ad-shGFP; HFG, HFD-fed ApoE KO mice treated with Ad-shFGF-21. Values are means $\pm \mathrm{SE}$.

${ }^{* *} P<0.01$ versus the NF group; ${ }^{\dagger} P<0.05,{ }^{\ddagger} P<0.01$ versus the HF group; ${ }^{\S} P<0.05,{ }^{\S} P<0.01$ versus the GFP group.

increased whole-body insulin resistance. The rate of glucose disappearance in HFD-fed mice (HF group) was lower than that in SCD-fed mice (NF group, $\sim 80 \%$, $P<0.01$ ), and administration of HFD plus Ad-sh $F G F$ 21 in the HFG group resulted in a further $5 \%$ decrease in the rate of disappearance of glucose compared with that in the GFP group $(P<0.05$, Fig. 3B). As expected, suppression of hepatic glucose production (HGP) by hyperinsulinemia was almost $70 \%$ in the NF group $\left(14.2 \pm 0.3\right.$ versus $4.3 \pm 2.3 \mathrm{mg} \cdot \mathrm{kg}^{-1} \cdot \mathrm{min}^{-1}$, Fig. 3C), 
Fig. 2. Steady-state plasma parameters during euglycemic/hyperinsulinemic clamp studies. (A) Experimental protocol for Ad-shFGF-21 delivery and insertion of venous and arterial catheters. (B) Experimental design for hyperinsulinemic/ euglycemic clamp studies. (C) Plasma insulin levels. (D) Plasma triglyceride levels. (E) Plasma total cholesterol levels. (F) Plasma free fatty acid levels. NF, SCDfed ApoE KO mice; HF, HFD-fed ApoE KO mice; GFP, HFD-fed ApoE KO mice treated with Ad-sh GFP; HFG, HFD-fed ApoE KO mice treated with Ad-shFGF-21. Values are means $\pm \mathrm{SE}$.
A

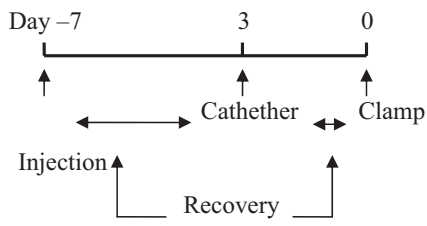

C

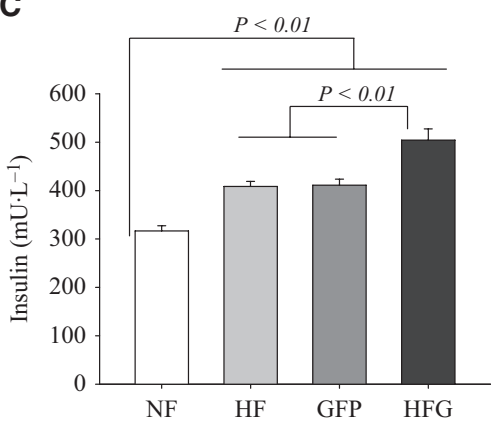

E

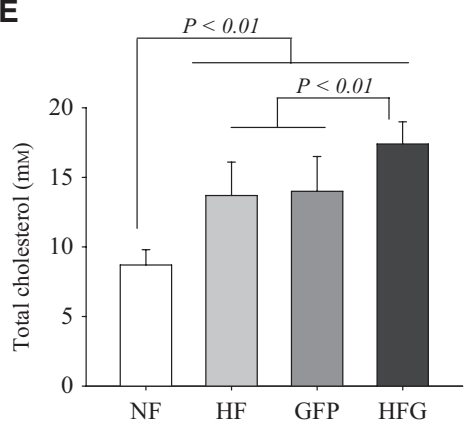

B

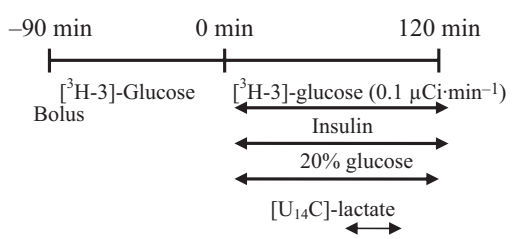

D

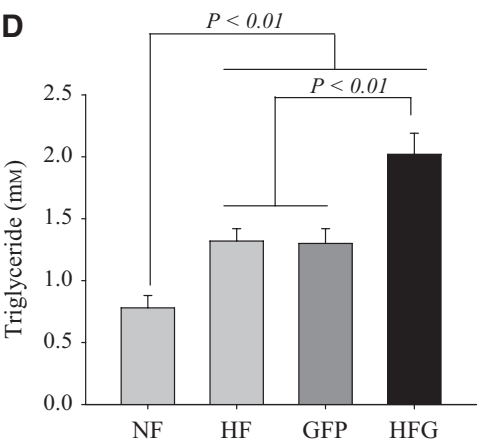

F

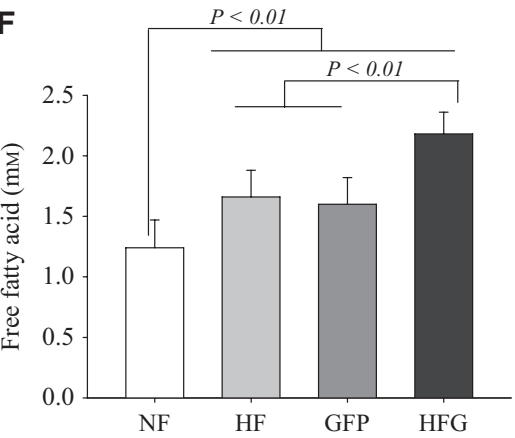

but only $\sim 51 \%$ in the HF group (16.2 \pm 0.3 versus $\left.8.0 \pm 5.5 \mathrm{mg} \cdot \mathrm{kg}^{-1} \cdot \mathrm{min}^{-1}\right)$. Importantly, in the HFG group, HGP was suppressed by only $20 \%$ (from $18.0 \pm 1.1$ to $14.4 \pm 2.4 \mathrm{mg} \cdot \mathrm{kg}^{-1} \cdot \mathrm{min}^{-1}$ ), indicating a marked increase in hepatic insulin resistance. The insulin action on glucose production was expressed as a percentage suppression of the basal levels (Fig. 3D). In addition, the GIR to insulin during the clamp was lowest in the HFG group, suggesting more serious insulin resistance in peripheral tissues of these animals $(P<0.05$ or $P<0.01$, Fig. 3E). The rate of glucose disappearance calculated on the basis of $\left[3-{ }^{3} \mathrm{H}\right]$ glucose radioactivity levels is shown in Fig. S2.

\section{Mechanisms by which FGF-21 knockdown increases glucose production}

To determine the mechanism by which FGF-21 knockdown modulates liver glucose homeostasis, we examined the in vivo glucose flux through G6Pase and the relative contribution of glucose cycling to G6Pase flux.
As shown in Fig. 4A,B, compared to SCD feeding (NF group), HFD feeding in the HF group increased the flux through G6Pase by $36 \%$ (from $7.8 \pm 1.1$ to $\left.10.6 \pm 1.3 \mathrm{mg} \cdot \mathrm{kg}^{-1} \cdot \mathrm{min}^{-1} ; \quad P<0.01\right)$ and glucose cycling by $45 \%$ (from $2.2 \pm 0.3$ to $3.2 \pm 0.5$ $\left.\mathrm{mg} \cdot \mathrm{kg}^{-1} \cdot \mathrm{min}^{-1} ; P<0.01\right)$. FGF-21 knockdown in the HFG group further increased the G6Pase flux by $32 \%$ (from $10.6 \pm 1.3$ to $13.8 \pm 1.0 \mathrm{mg} \cdot \mathrm{kg}^{-1} \cdot \mathrm{min}^{-1}$, $P<0.05$ ) and glucose cycling by $28 \%$ (from $3.2 \pm 0.5$ to $4.1 \pm 0.6 \mathrm{mg} \cdot \mathrm{kg}^{-1} \cdot \mathrm{min}^{-1}, P<0.05$, Fig. 4 A,B), suggesting that FGF-21 knockdown increased the activity of G6Pase in the livers of HFD mice. We next examined the effect of FGF-21 knockdown on the relative contribution of glycogenolysis and gluconeogenesis to G6Pase flux. The effects of HFD feeding on glucose production and G6Pase flux in mice were accounted by a $25 \%$ increase in the rate of glycogenolysis $\left(2.0 \pm 0.5 \mathrm{mg} \cdot \mathrm{kg}^{-1} \cdot \mathrm{min}^{-1}\right.$ in the HF group versus $1.3 \pm 0.4 \mathrm{mg} \cdot \mathrm{kg}^{-1} \cdot \mathrm{min}^{-1}$ in the NF group, $P<0.05$ ) and a $75 \%$ increase in the rate of gluconeogenesis $\left(6.0 \pm 1.6 \mathrm{mg} \cdot \mathrm{kg}^{-1} \cdot \mathrm{min}^{-1}\right.$ in the $\mathrm{HF}$ group versus 

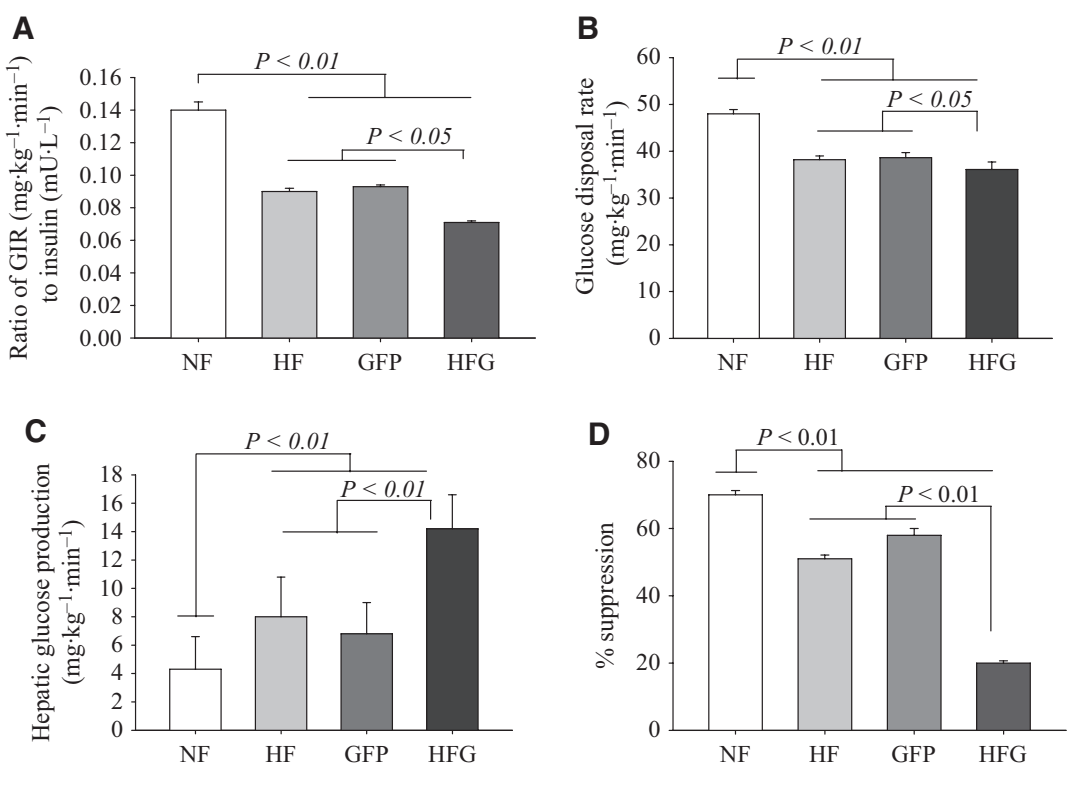

Fig. 3. Systemic administration of AdshFGF-21 increases insulin resistance in HFD-fed ApoE KO mice. (A) Glucose infusion rate $(G I R)$ required to maintain euglycemia during the clamp period. (B) Glucose disappearance rate during the clamp period. (C) Hepatic glucose production during the clamp period. (D) Suppression of glucose production during the clamp period expressed as the percentage decrease from basal glucose production. (E) Ratio of glucose infusion rate $(\mathrm{GIR})\left(\mathrm{mg} \cdot \mathrm{kg}^{-1} \cdot \mathrm{min}^{-1}\right)$ to insulin $\left(\mathrm{mU} \cdot \mathrm{L}^{-1} \cdot \mathrm{min}^{-1}\right)$ during the clamp period. NF, SCD-fed ApoE KO mice; HF, HFD-fed ApoE KO mice; GFP, HFD-fed ApoE KO mice treated with Ad-sh GFP; HFG, HFDfed ApoE KO mice treated with Ad-shFGF21. Values are means \pm SE.

$3.0 \pm 0.6 \mathrm{mg} \cdot \mathrm{kg} \cdot \mathrm{min}^{-1}$ in the NF group, $\left.P<0.01\right)$. In the HFG group, the increase in HGP by FGF-21 knockdown was accounted for by a $39 \%$ increase in the rate of glycogenolysis $\left(5.6 \pm 1.1 \mathrm{mg} \cdot \mathrm{kg}^{-1} \cdot \mathrm{min}^{-1}\right)$ and a $61 \%$ increase in the rate of gluconeogenesis $\left(8.8 \pm 1.2 \mathrm{mg} \cdot \mathrm{kg}^{-1} \cdot \mathrm{min}^{-1}\right)$ (Fig. 4C,D). Furthermore, hepatic glycogen in the HFG group was significantly higher than that in the HF and GFP groups $(P<0.05$, Table 1).

In agreement with changes in glucose metabolism, the FGF-21 knockdown in the HFG group significantly increased hepatic G6Pase mRNA and protein expression compared with in other three groups (Fig. 5A,C). Moreover, FGF-21 knockdown in the HFG group also resulted in a marked increase in phosphoenolpyruvate carboxykinase (PEPCK) mRNA and protein expression (Fig. 5B,D). Hepatic mRNA expression of pyruvate carboxylase was significantly lower in the NF group than in the HF and GFP groups. However, hepatic pyruvate carboxylase mRNA levels in the HFG group were similar to those in the HF and GFP groups (Fig. 5E).

\section{Effects of FGF-21 knockdown on the STAT3/ SOCS3 pathway}

Recent in vivo studies have shown that STAT3 directly targets the regulatory regions of both G6Pase and PEPCK [17,19]. To determine whether the effect of FGF-21 knockdown on insulin sensitivity was associated with changes in STAT3 signaling, we examined the activation of STAT3 in the liver by western blot analysis. HFD feeding in the HF and GFP groups reduced phosphorylation of STAT3 in the liver compared with SCD feeding in the NF group, and FGF-21 knockdown in the HFG group further promoted this reduction, suggesting that FGF-21 may regulate STAT3 activation by directly phosphorylating its tyrosine residues (Fig. 6A). Because one of the transcriptional targets of STAT3 is SOCS3 [20], we examined the SOCS3 mRNA and protein levels in hepatic tissues of mice. As shown in Fig. 6B,C, SOCS3 mRNA and protein levels in the HFG group were decreased to $\sim 22 \%$ and $43 \%$ respectively, compared to the HF and GFP groups $(P<0.05)$. 
A

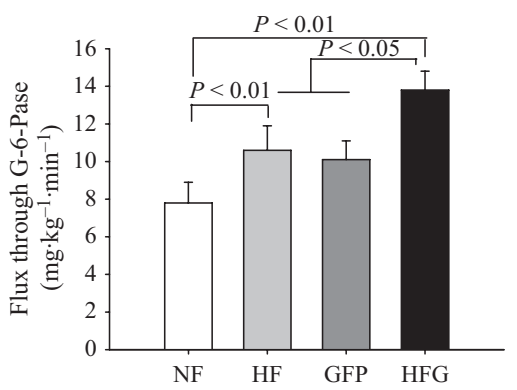

Fig. 4. Effects of FGF-21 knockdown on liver glucose fluxes. (A) In vivo flux of glucose through G6Pase. (B) Glucose cycling (the futile cycle between glucokinase and G6Pase). (C) Rate of glycogenolysis. (D) Rate of gluconeogenesis. NF, SCD-fed ApoE KO mice; HF, HFD-fed ApoE KO mice; GFP, HFD-fed ApoE KO mice treated with AdshGFP; HFG, HFD-fed ApoE KO mice treated with Ad-shFGF-21. Values are means \pm SE.

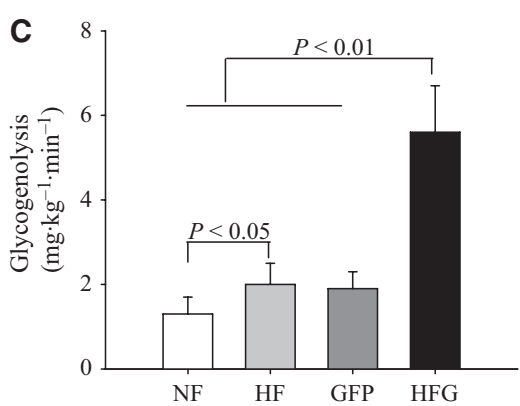

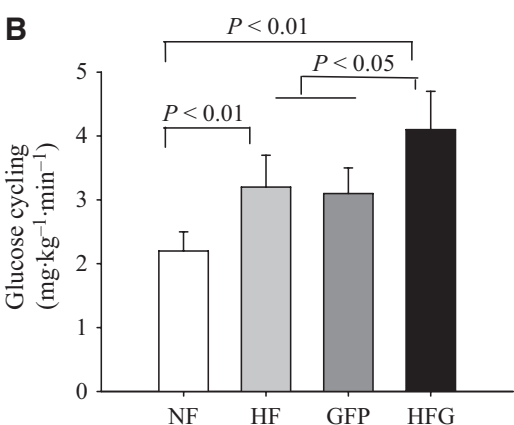



\section{Discussion}

Recent reports have shown that FGF-21 participates in the regulation of glucose homeostasis [21]. The lack of mitogenic action makes this molecule an attractive candidate to treat type 2 diabetes and related metabolic diseases. In a previous study, we showed that plasma FGF-21 concentrations were significantly increased in diabetic subjects [1]. Because diet-induced obese mice have increased endogenous levels of FGF-21 and respond poorly to exogenous FGF-21 [6], the elevation of circulating FGF-21 in the subjects with type 2 diabetes may be a compensatory response to the metabolic stress imposed by obesity or type 2 diabetes. This phenomenon is similar to hyperinsulinemia and hyperleptinemia, in which the increased insulin and leptin production is thought to compensate for the insulin and leptin resistance associated with obesity.

Although FGF-21 has been reported to affect glucose metabolism and insulin action, its effects on glucose kinetics and insulin signaling, particularly the STAT3/SOCS3 pathway, have not been systematically examined. In this study, we have developed a mouse model comprising adenovirus-mediated shRNA knockdown of hepatic FGF-21. We demonstrated that use of adenovirus-mediated shRNA against FGF-21 resulted in silencing of FGF-21 and some long-term effects (up to 2 weeks), which allowed us to study the metabolic impact of hepatic FGF-21 knockdown in an insulin- resistance model (HFD-fed ApoE KO mice).
ApoE KO mice that develop severe hypercholesterolemia and atherosclerosis on a SCD $[22,23]$ are widely used as a model for HFD-induced insulin resistance $[13,23,24]$. In our study, the mice with FGF-21 knockdown showed significant increases in blood glucose, plasma insulin, FFA, lipid levels and hepatic TG compared to controls. In addition, their GIR, an index of glucose utilization, was decreased, whereas their HGP was increased. Therefore, selective inhibition of FGF21 in the liver was sufficient to inhibit the effect of insulin on lipolysis and glucose production. Furthermore, FGF-21 knockdown in Ad-shFGF-21-treated mice led to a further decrease in the GIR, suggesting lower glucose uptake and more serious insulin resistance in the peripheral tissues of these animals.

We also examined the effect of FGF-21 knockdown on glucose flux through G6Pase, the most distal step in HGP. We found that, in HFD-fed mice, FGF-21 knockdown increased glucose flux through G6Pase and glucose cycling. More importantly, we demonstrated that the FGF-21 knockdown-mediated increase in glucose production and G6Pase flux was due to increases in glycogenolysis and gluconeogenesis. Consistent with these results, FGF-21 knockdown markedly increased hepatic G6Pase and PEPCK mRNA and protein levels. However, FGF-21 knockdown had little effect on the expression of pyruvate carboxylase, another gluconeogenetic enzyme that catalyzes the carboxylation of pyruvate to oxaloacetate [25]. Pyruvate carboxylase is exclusively expressed in mitochondria 

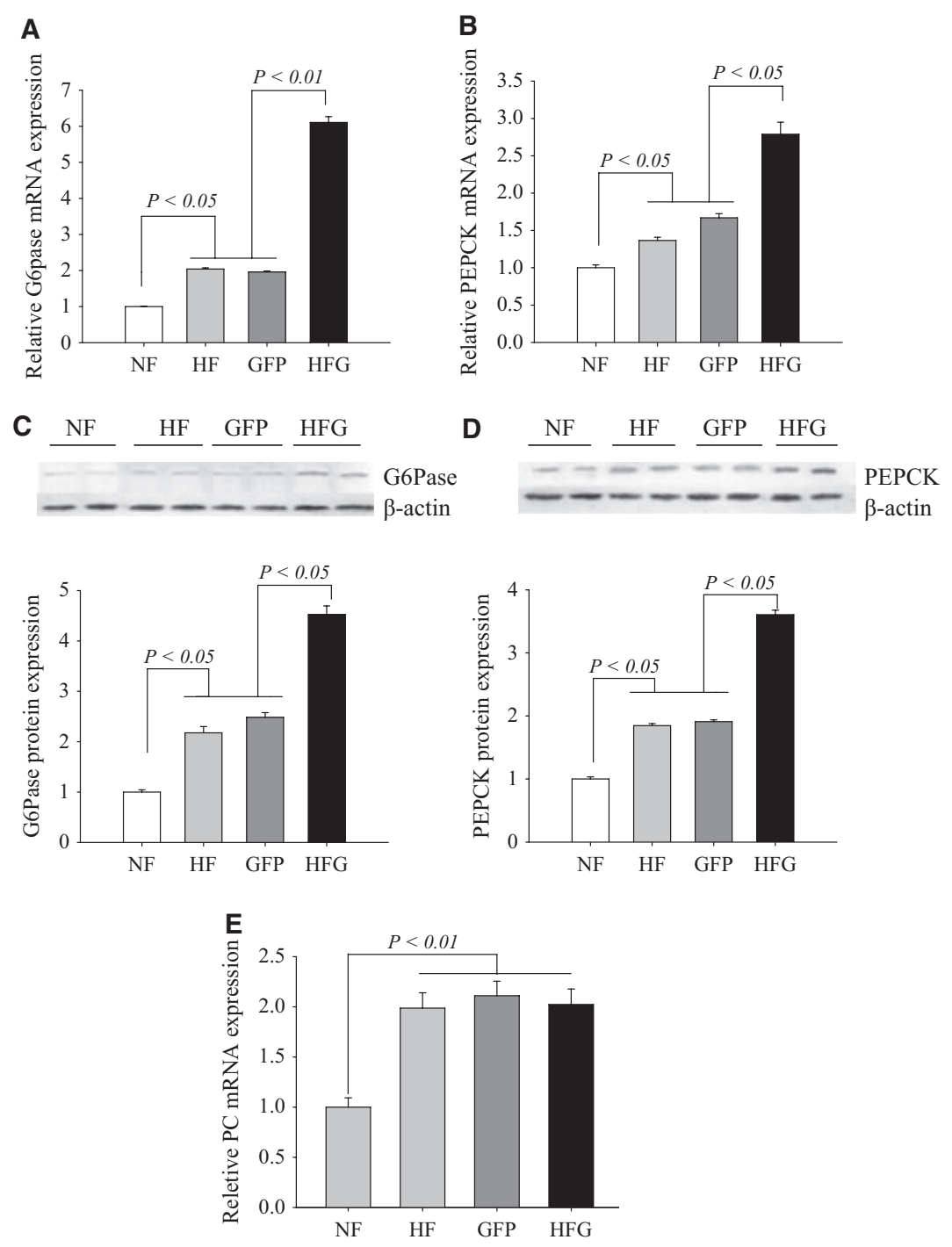

Fig. 5. mRNA and protein expression of PEPCK and G6Pase in the liver. Total protein and RNA were extracted from frozen liver for the various groups. Western blots and quantitative real-time PCR were performed using pooled protein and RNA extracts. (A) Relative G6Pase mRNA expression. (B) Relative PEPCK mRNA expression. (C) G6Pase protein abundance. (D) PEPCK protein abundance. (E) Relative pyruvate carboxylase (PC) mRNA expression. NF, SCD-fed ApoE KO mice; HF, HFD-fed ApoE KO mice; GFP, HFD-fed ApoE KO mice treated with AdshGFP; HFG, HFD-fed ApoE KO mice treated with Ad-shFGF-21. Values are means \pm SE from five independent experiments, and are expressed as fold changes relative to the levels in the NF group.

and contributes to de novo fatty acid synthesis by providing oxaloacetate for conversion to citrate, which is exported from the mitochondria and cleaved in the cytosol to form oxaloacetate and acetyl CoA [26]. Thus, pyruvate carboxylase is apparently not essential for gluconeogenesis. Therefore, it appears that the effect of FGF-21 knockdown on gluconeogenesis is mainly due to increased activation of hepatic G6Pase and PEPCK.

It has previously been reported that STAT3 plays a role in suppressing expression of gluconeogenic genes $[19,27]$. To investigate the role of hepatic STAT3 signaling in mediating the effect of FGF-21 knockdown on HGP, we examined hepatic STAT3 phosphorylation in HFD-fed mice with FGF-21 knockdown. We found that FGF-21 knockdown decreased the hepatic phosphorylation of STAT3 in HFD-fed mice. This finding provides support for the notion that FGF-21 knockdown modulates hepatic glucose homeostasis via the STAT3 pathway, leading to hepatic activation of G6Pase and PEPCK, and increased gluconeogenesis and glycogenolysis. Our results are consistent with previous findings showing that STAT3 negatively regulates gluconeogenic gene expression in vivo [17].

Recent studies have shown that SOCS3 is regulated by the STAT pathway and various cytokines and hormones, such as tumor necrosis factor $\alpha$, interleukin-6, resistin and adiponectin, all of which affect insulin sensitivity $[28,29]$. It has also been reported that SOCS3 plays a role in the pathogenesis of insulin resistance by integrating cytokine signaling with insulin signaling [20]. To determine whether the insulin-desensitizing effect of FGF-21 knockdown is related to SOCS3 signaling, we examined the effect of FGF-21 knockdown 
Fig. 6. Effects of FGF-21 knockdown on liver SOCS3 expression and STAT3 activation. Total protein and RNA were extracted from liver samples of mice. Western blotting and real-time PCR analyses were performed on pooled protein and RNA extracts. (A) STAT3 phosphorylation. (B) Relative SOCS3 mRNA expression. (C) SOCS3 protein abundance. NF, SCD-fed ApoE KO mice: HF, HFD-fed ApoE KO mice; GFP, HFDfed ApoE KO mice treated with Ad-sh GFP; HFG, HFD-fed ApoE KO mice treated with Ad-shFGF-21. Values are means \pm SE from five independent experiments, and are expressed as fold changes relative to the levels in the NF group.
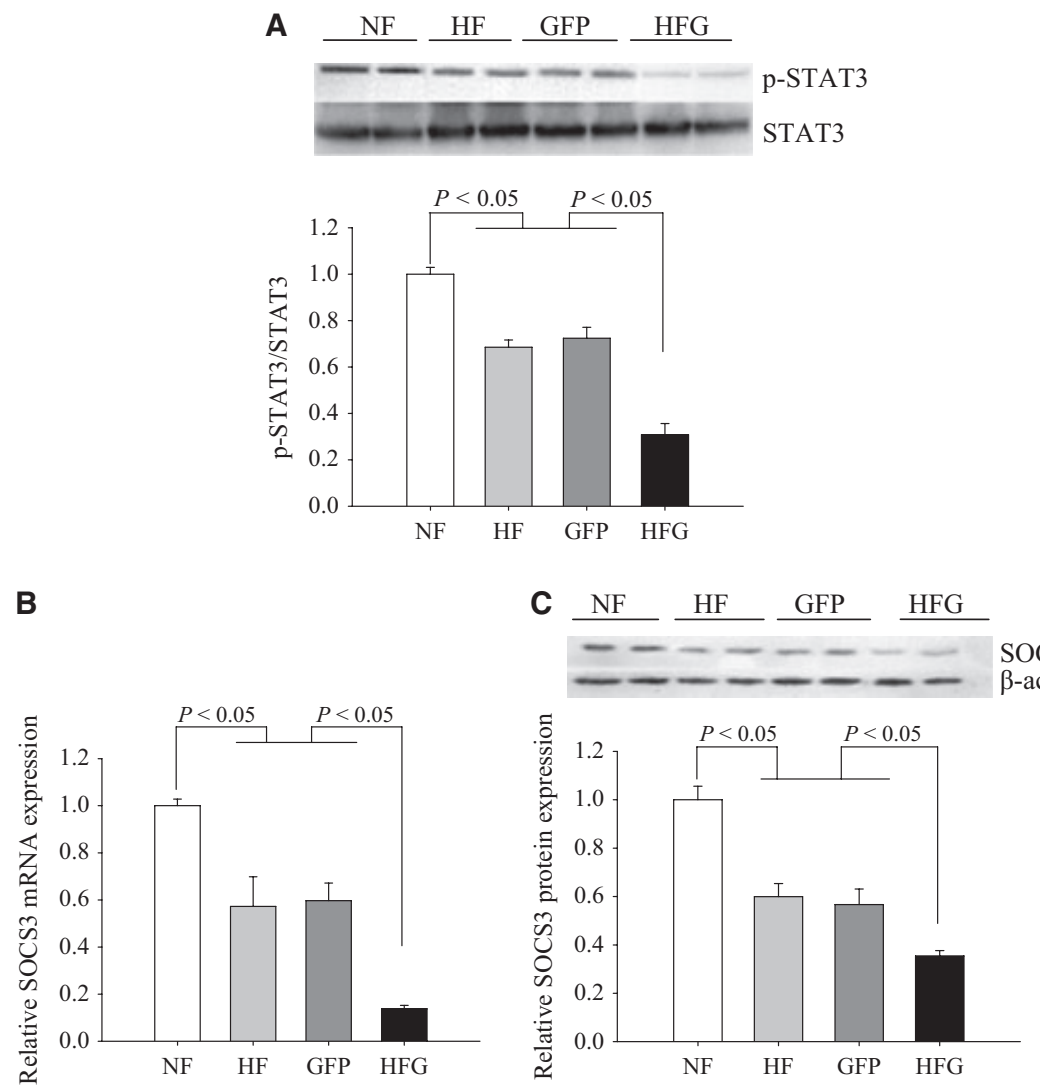

on SOCS3 expression in the liver. FGF-21 knockdown induced decreases in SOCS3 mRNA and protein levels in the liver of HFD-fed mice, associated with decreased STAT3 activation and increased insulin resistance. To date, the role of SOCS3 in insulin resistance has remained controversial. Some studies have reported a negative feedback relationship between SOCS3 and insulin signaling (e.g. over-expression of SOCS3 in liver caused insulin resistance, whereas suppression of SOCS3 expression ameliorated insulin resistance) [16,30], and others have shown a positive relationship between SOCS3 and insulin action [31]. In addition, over-expression of SOCS3 in adipose tissue has been shown to inhibit insulin action but to systematically improve glucose metabolism under HFD conditions [32]. In agreement with a previous study [31], our results showed that the decrease of SOCS3 induced by FGF-21 knockdown in the liver increased insulin resistance. However, it should also be noted that the relationship between STAT3 and SOCS3 remains controversial. Ramadoss et al. [17] reported that STAT3 serves as a positive regulator of SOCS3 expression, whereas others have shown that STAT3 is a negative regulator of SOCS3 expression [16,29]. In agreement with several previous studies, our results showed that a decrease in STAT3 phosphorylation accompanied by a decrease in SOCS3 expression, induced by FGF-21 knockdown in the liver, further promoted insulin resistance.

In conclusion, our findings suggest that FGF-21 knockdown in the liver increases gluconeogenesis and glycogenolysis by activation of G6Pase and PEPCK via the STAT3/SOCS3 pathway. This leads to exacerbated hepatic insulin resistance in HFD-fed ApoE KO mice. These results provide in vivo evidence that the insulin-sensitizing role of FGF-21 with respect to changes in glucose flux is linked to the STAT3/SOCS3 pathway.

\section{Experimental procedures}

\section{Construction and purification of adenoviruses expressing shRNA against FGF-21}

The adenoviral vectors that express shRNA against FGF-21 were constructed as previously described [33,34]. The pAdxsi system (SinoGenoMax Co. Ltd, Beijing, China) was used for shRNA construction. Briefly, two oligonucleotides and complementary strands specific to mouse FGF-21 were synthesized. The sequences were as follows: 
sense 5'-TTTGGGGTCTACCAAGCATACCCCATTCA AGACGTGGGGTATGCTTGGTAGACCCTTTTTTG-3' and antisense 5'-AGCTCAAAAAAGGGTCTACCAAG CATACCCCACGTCTTGAATGGGGTATGCTTGGTAGA -CCC- $3^{\prime}$. First, the annealed oligonucleotides were ligated into the pSilencer1.2-U6 vector (Stratagene, Santa Clara, CA, USA) and their sequences were verified. FGF-21 shRNA expression plasmids were transfected into 3T3-L adipocytes, which were harvested $48 \mathrm{~h}$ later to test the efficiency of these shRNA for FGF-21 knockdown. Second, the selected pSilencer1.0-U6-shRNA plasmids were recombined into the Gateway-based pAdxsi vector (Ad-shFGF21) to generate recombinant adenovirus vectors expressing shRNA for FGF-21. A recombinant adenovirus vector that expresses an shRNA against GFP (Ad-shGFP) was used as a negative control. Finally, the recombinant adenoviruses were amplified using HEK 293A cells, according to the manufacturer's instructions (Stratagene adenovirus amplification kit, Stratagene), and a ViraBind adenovirus purification kit (Cell Biolabs Inc., San Diego, CA, USA) was used for large-scale amplification and purification of recombinant adenoviruses according to the manufacturer's instructions [35].

\section{Mouse models and administration of adenoviruses}

To establish the insulin resistance model, male ApoE KO mice at 3 weeks of age were obtained from the Experimental Animal Center of Beijing University of Medical Sciences (Beijing, China), acclimatized for a week, and fed with either SCD (18\% kcal from fat) or HFD (54\% kcal from fat; Research Diets Inc., New Brunswick, NJ, USA) for 16 weeks. Each mouse was separately housed in a temperature-controlled $\left(24{ }^{\circ} \mathrm{C}\right)$ facility with a $12 \mathrm{~h}$ light $/ 12 \mathrm{~h}$ dark cycle (lights on at 22:00 h). SCD-fed ApoE KO mice (NF group, $n=10$ ) were used as diet controls. Twenty-six HFDfed ApoE KO mice were further divided into three groups, and treated with $100 \mu \mathrm{L}$ Ad-shFGF-21 $\left(1 \times 10^{9}\right.$ plaqueforming units $\mathrm{g}^{-1}$ ) (HFG group, $\left.n=10\right)$, Ad-shGFP (GFP group, $n=6$ ) or sterile saline (HF group, $n=10$ ). The adenoviruses expressing shGFP or $\operatorname{sh} F G F-21$ were injected into mice through a tail vein at the end of both 14th and 15th weeks of HFD feeding (Fig. 3A). As previously described [36,37], 4 days before performing clamp experiments, the mice were catheterized through the right jugular vein and the left carotid artery under pentobarbital sedation $\left(50 \mathrm{mg} \cdot \mathrm{kg}^{-1}\right.$; Nembutal Abbott Laboratories, Abbott Park, IL, USA). Catheters were placed subcutaneously, attached at the back of the neck, and filled with heparinized saline. The jugular and carotid catheters were used for blood sampling and infusion, respectively. All studies were performed in accordance with the guidelines for the use and care of laboratory animals of the Chongqing Medical University Institutional Animal Care and Use Committee.

\section{Hyperinsulinemic/euglycemic clamps}

To assess the effect of euglycemia/hyperinsulinemia on glucose turnover, euglycemic/hyperinsulinemic clamps were performed for $2 \mathrm{~h}$ after 7 days of adenoviral administration as described previously [34,36]. Briefly, mice under chronically cannulated, conscious and unrestrained conditions were fasted for $12 \mathrm{~h}$ before the studies. A bolus of insulin $\left(16 \mathrm{mU} \mathrm{kg}{ }^{-1}\right)$ and $\left[3-{ }^{3} \mathrm{H}\right]$ glucose $\left(5 \mu \mathrm{Ci} \cdot \mathrm{kg}^{-1}\right)$ was injected, and thereafter insulin (Novo R, Novo Nordisk, Bagsvaerd, Denmark, $5 \mathrm{mU} \cdot \mathrm{kg}^{-1} \cdot \mathrm{min}^{-1}$ ) was infused at a constant rate throughout the clamp. $\left[3-{ }^{3} \mathrm{H}\right]$-glucose was concomitantly infused with insulin and $25 \%$ glucose via the carotid catheter at a rate of $0.1 \mu \mathrm{Ci} \cdot \mathrm{kg}^{-1} \cdot \mathrm{min}^{-1}$. Blood was sampled from the jugular venous catheter every $10 \mathrm{~min}$ to determine blood glucose concentration and to adjust the glucose infusion rate to maintain blood glucose at its initial basal level in each group. To determine the contribution of gluconeogenesis to the pool of hepatic glucose-6-phosphate, $\left[\mathrm{U}_{-}{ }^{14} \mathrm{C}\right]-$ lactate (New England Nuclear, Boston, MA, USA; $20 \mu \mathrm{Ci}$ bolus; $1.0 \mu \mathrm{Ci} \cdot \mathrm{min}^{-1}$ ) was administered to mice $10 \mathrm{~min}$ before the end of the infusions. For determination of plasma $\left[3-{ }^{3} \mathrm{H}\right]$ glucose concentrations, blood samples $(20 \mu \mathrm{L})$ were collected at 80, 90, 100 and 120 min after starting the clamp experiments. For measurement of plasma glucose, insulin, TG, TC, LDL-C, HDL-C and FFA, additional blood samples $(50 \mu \mathrm{L})$ were collected before the start and at the end of the clamps. The ratio of the glucose infusion rate to insulin was calculated as an insulin sensitivity index in peripheral tissues. After completion of the clamp experiments, mice were anesthetized with a sodium pentobarbital injection, the abdomen was quickly opened, and portal vein blood was collected. Within $3 \mathrm{~min}$, hepatic tissues were harvested, frozen immediately using liquid nitrogen-cooled aluminum blocks, and stored at $-80{ }^{\circ} \mathrm{C}$ for subsequent analysis.

\section{Analytical procedures}

For determination of plasma $\left[3-{ }^{3} \mathrm{H}\right]$ glucose, plasma was deproteinized with $\mathrm{ZnSO}_{4}$ and $\mathrm{Ba}(\mathrm{OH})_{2}$, dried to remove $\mathrm{H}_{2} \mathrm{O}$, resuspended in water, and counted in scintillation fluid (Ultima Gold; Packard Instrument Co., Meriden, CT, USA). After HPLC measurement of hepatic $\left[{ }^{14} \mathrm{C}\right]$-phosphoenolpyruvate and $\left[{ }^{3} \mathrm{H} /{ }^{14} \mathrm{C}\right]$-UDP-glucose specific activities the rates of phosphoenolpyruvate gluconeogenesis were calculated. The ratio of liver $\left[{ }^{3} \mathrm{H}\right]$-UDP-glucose and plasma $\left[3-{ }^{3} \mathrm{H}\right]$-glucose-specific activities represents the percentage of the hepatic glucose-6-phosphate pool directly derived from plasma glucose (direct pathway). Gluconeogenesis was determined from the specific activities of ${ }^{14} \mathrm{C}$-labeled hepatic UDP-glucose (assumed to reflect the specific activity of hepatic glucose-6-phosphate) and hepatic phosphoenolpyruvate following infusion of $\left[\mathrm{U}-{ }^{14} \mathrm{C}\right]$-lactate and $\left[3-{ }^{3} \mathrm{H}\right]$-glucose using the formula: gluconeogenesis = total glucose 
output $\times\left[{ }^{14} \mathrm{C}\right]$-UDP-glucose specific activity/[ $\left.{ }^{14} \mathrm{C}\right]$-phosphoenolpyruvate specific activity $\times 2$, as described elsewhere [35]. Plasma insulin levels were determined using a commercial insulin enzyme-linked immunosorbent assay kit (Diagnostic Products, Los Angeles, CA, USA), and the plasma FFA level was determined spectrophotometrically using an acyl CoA oxidase-based colorimetric kit (Wako Pure Chemical Industries, Osaka, Japan) [38]. The glucose oxidase method was used for plasma glucose measurement. Plasma TG, TC, HDL-C and LDL-C concentrations were measured by the enzymatic colorimetric method (Sigma, St Louis, MO, USA). Plasma FGF-21 levels were determined using an ELISA kit (Phoenix Pharmaceuticals, Belmont, CA, USA). Hepatic TG and glycogen levels were measured by a spectrophotometric procedure as described previously $[39,40]$.

\section{Quantitative real-time PCR}

Quantitative real-time PCR was performed as described previously [33]. Briefly, muscle and hepatic tissue RNA was isolated from frozen tissue (100 mg) using Trizol reagent (Invitrogen, Carlsbad, CA, USA). Purified total RNAs were used for cDNA synthesis using a PrimerScript ${ }^{\mathrm{TM}}$ reverse transcription reagent kit (Takara Bio Inc., Otsu, Japan). Quantitative real-time PCR was performed using a SYBR Green PCR kit (Takara Bio Inc.) and a Corbett Rotor Gene 6000 real-time PCR system (Corbett Research, Sydney, Australia). Expression of genes was analyzed by the comparative $C_{\mathrm{t}}$ method, and normalized against that of $\beta$-actin. The primer pairs used for quantitative real-time PCR are listed in Table S1.

\section{Western blot analysis}

Western blot analysis was performed as described previously [35]. Briefly, liver was homogenized in a solution of $20 \mathrm{~mm}$ MOPS, 2 mм EGTA, 5 mм EDTA, 30 mm sodium fluoride, $40 \mathrm{~mm} \beta$-glycerophosphate, $10 \mathrm{~mm}$ sodium pyrophosphate, $2 \mathrm{~mm}$ orthovanadate, $0.5 \% \mathrm{NP}-40$, and complete phosphatase inhibitor cocktail (Roche). Protein concentration was determined using a BCA protein quantification kit (Pierce Biotechnology, Rockford, IL, USA). Extracted proteins were separated on either 4-12\% Criterion XL (Bio-Rad, Hercules, CA, USA) or 4-12\% Bis-Tris NuPAGE (Invitrogen) gels, and blotted onto Immobilon FL poly(vinylidene difluoride) membranes (Millipore, Billerica, MA, USA). The films were blocked at room temperature for $1 \mathrm{~h}$ in Odyssey blocking buffer (LI-COR Biosciences, Lincoln, NE, USA) and incubated with primary antibodies against p-STAT3705 (Cell Signaling Technology, Beverly, MA, USA), STAT3 (Santa Cruz Biotechnology Inc., Dallas, TX, USA), FGF-21, SOCS3, G6Pase, PEPCK and $\beta$-actin (Research Diagnostics Inc.) diluted $1: 1000$ in blocking buffer (Tris-buffered saline/Tween-20) at $4{ }^{\circ} \mathrm{C}$ overnight. After three washes with Tris-buffered saline/Tween-20 (0.1\%), the blots were incubated with horseradish peroxidase-conjugated secondary antibodies (Invitrogen) (1:500 dilution) for $1 \mathrm{~h}$ at room temperature. After two washes with Tris-buffered saline/Tween-20 and a final wash with Tris-buffered saline for 10 minutes at room temperature every time, the blots were scanned using the Odyssey infrared imaging system (LI-COR Biosciences), and the antigen-antibody complexes were quantified using QUANTITY ONE analysis software (BioRad) as described previously [33].

\section{Statistical analysis}

Data are expressed as means \pm SE. Comparisons among groups were performed by ANOVA, followed by a post hoc least-squares difference test. Differences at $P<0.05$ were considered statistically significant. All data were statistically analyzed using SPSS 13.0 (SPSS, Chicago, IL, USA).

\section{Acknowledgements}

This work was supported by research grants from the National Natural Science Foundation of China (81270913, 81070640, 81100567, 81300702, 81100567 and 81300670), the Doctoral Fund of the Ministry of Education of China (20105503110002 and 2012550 3110003), the Natural Science Foundation Key Project of CQ CSTC (cstc2012, jjB10022), and an American Diabetes Association grant (number 1-10-CT-06) to G.B.

\section{References}

1 Chen WW, Li L, Yang GY, Li K, Qi XY, Zhu W, Tang Y, Liu H \& Boden G (2007) Circulating FGF-21 levels in normal subjects and in newly diagnosed patients with type 2 diabetes mellitus. Exp Clin Endocrinol Diabetes 115, 1-4.

2 Li L, Yang G, Ning H, Yang M, Liu H \& Chen W (2008) Plasma FGF-21 levels in type 2 diabetic patients with ketosis. Diabetes Res Clin Pract 82, 209-213.

3 Li K, Li L, Yang M, Zong H, Liu H \& Yang G (2009) Effects of rosiglitazone on fasting plasma fibroblast growth factor-21 levels in patients with type 2 diabetes mellitus. Eur J Endocrinol 161, 391-395.

4 Muise ES, Azzolina B, Kuo DW, El-Sherbeini M, Tan Y, Yuan X, Mu J, Thompson JR, Berger JP \& Wong KK (2008) Adipose fibroblast growth factor 21 is upregulated by peroxisome proliferator-activated receptor $\gamma$ and altered metabolic states. Mol Pharmacol 74, 403-412.

5 Zhang X, Yeung DC, Karpisek M, Stejskal D, Zhou ZG, Liu F, Wong RL, Chow WS, Tso AW, Lam KS, et al. (2008) Serum FGF21 levels are increased in 
obesity and are independently associated with the metabolic syndrome in humans. Diabetes 57, 1246-1253.

6 Fisher MF, Chui PC, Antonellis PJ, Bina HA, Kharitonenkov A, Flier JS \& Maratos- Flier E (2010) Obesity is a fibroblast growth factor 21 (FGF21)resistant state. Diabetes 59, 2781-2789.

7 Kharitonenkov A, Shiyanova TL, Koester A, Ford AM, Micanovic R, Galbreath EJ, Sandusky GE, Hammond LJ, Moyers JS, Owens RA, et al. (2005) FGF-21 as a novel metabolic regulator. $J$ Clin Invest 115, 1627-1635.

8 Coskun T, Bina HA, Schneider MA, Dunbar JD, Hu CC, Chen Y, Moller DE \& Kharitonenkov A (2008) Fibroblast growth factor 21 corrects obesity in mice. Endocrinology 149, 6018-6027.

9 Xu J, Lloyd DJ, Hale C, Stanislaus S, Chen M, Sivits G, Vonderfecht S, Hecht R, Li YS, Lindberg RA, et al. (2009) Fibroblast growth factor 21 reverses hepatic steatosis, increases energy expenditure, and improves insulin sensitivity in diet-induced obese mice. Diabetes 58, 250-259.

10 Kharitonenkov A, Wroblewski VJ, Koester A, Chen YF, Clutinger CK, Tigno XT, Hansen BC, Shanafelt AB \& Etgen GJ (2007) The metabolic state of diabetic monkeys is regulated by fibroblast growth factor- 21 . Endocrinology 148, 774-781.

11 Camporez JP, Jornayvaz FR, Petersen MC, Pesta D, Guigni BA, Serr J, Zhang D, Kahn M, Samuel VT, Jurczak MJ, et al. (2013) Cellular mechanisms by which FGF21 improves insulin sensitivity in male mice. Endocrinology 154, 3099-3109.

12 Gable D, Sanderson SC \& Humphries SE (2007) Genotypes, obesity and type 2 diabetes - can genetic information motivate weight loss? A review. Clin Chem Lab Med 45, 301-308.

13 Hansmann G, Wagner RA, Schellong S, Perez VA \& Urashima T (2007) Pulmonary arterial hypertension is linked to insulin resistance and reversed by peroxisome proliferator-activated receptor- $\gamma$ activation. Circulation 115, 1275-1284.

14 To AW, Ribe EM, Chuang TT, Schroeder JE \& Lovestone $S$ (2011) The $\varepsilon 3$ and $\varepsilon 4$ alleles of human APOE differentially affect tau phosphorylation in hyperinsulinemic and pioglitazone-treated mice. PLoS ONE 6, e16991.

15 Li L, Miao Z, Liu R, Yang M, Liu H \& Yang G (2011) Liraglutide prevents hypoadiponectinemiainduced insulin resistance and alterations of gene expression involved in glucose and lipid metabolism. Mol Med 17, 1168-1178.

16 Kanatani Y, Usui I, Ishizuka K, Bukhari A, Fujisaka S, Urakaze M, Haruta T, Kishimoto T, Naka T \& Kobayashi M (2007) Effects of pioglitazone on suppressor of cytokine signaling 3 expression. Diabetes 56, 795-803.
17 Ramadoss P, Unger-Smith NE, Lam FS \& Hollenberg AN (2009) STAT3 targets the regulatory regions of gluconeogenic genes in vivo. Mol Endocrinol 23, 827837.

18 Ling L, Gangyi Y, Shaochuan S, Mengliu Y, Hua L \& Boden G (2012) The effects of fibroblast growth factor21 knockdown and over-expression on its signaling pathway and glucose-lipid metabolism in vitro. Mol Cell Endocrinol 348, 21-26.

19 Inoue H, Ogawa W, Ozaki M, Haga S, Matsumoto M, Furukawa K, Hashimoto N, Kido Y, Mori T, Sakaue $\mathrm{H}$, et al. (2004) Role of STAT-3 in regulation of hepatic gluconeogenic genes and carbohydrate metabolism in vivo. Nat Med 10, 168-174.

20 Howard JK, Flier JS, Howard JK \& Flier JS (2006) Attenuation of leptin and insulin signaling by SOCS proteins. Trends Endocrinol Metab 17, 365-371.

21 Moller DE (2001) New drug targets for type 2 diabetes and the metabolic syndrome. Nature 414, 821-827.

22 Breslow JL (1996) Mouse models of atherosclerosis. Science 272, 685-688.

23 Zhang SH, Reddick RL, Piedrahita JA \& Maeda N (1992) Spontaneous hypercholesterolemia and arterial lesions in mice lacking apolipoprotein E. Science 258, 468-471.

24 Chen Y, Yang G, Li L, Li K \& Chen W (2009) The changes of glucose-lipid metabolism in ApoE-/- mice with high-fat induced insulin resistance. Chin J Diabetes 17, 590-592.

25 Utter MF \& Keech DB (1963) Pyruvate carboxylase. I. Nature of the reaction. J Biol Chem 238, 2603-2608.

26 Ballard FJ \& Hanson RW (1967) The citrate cleavage pathway and lipogenesis in rat adipose tissue: replenishment of oxaloacetate. J Lipid Res 8, 73-79.

27 Inoue H, Ogawa W, Asakawa A, Okamoto Y, Nishizawa A, Matsumoto M, Teshigawara K, Matsuki Y, Watanabe E, Hiramatsu R, et al. (2006) Role of hepatic STAT3 in brain-insulin action on hepatic glucose production. Cell Metab 8, 3267-3275.

28 Uno T, He T, Usui I, Kanatani Y, Bukhari A, Fujisaka $\mathrm{S}$, Yamazaki Y, Suzuki H, Iwata M, Ishiki M, et al. (2008) Long-term interleukin- $1 \alpha$ treatment inhibits insulin signaling via IL-6 production and SOCS3 expression in 3T3-L1 adipocytes. Horm Metab Res 40, $8-12$.

29 Run-Zhe S, Feng Z, Fang W, De-Chun F, Xi-Hua L, Wei-Hua R, Xiao-Lin W, Xue Y, Xiao-Dong L, Lei H, et al. (2009) Adiponectin deficiency impairs liver regeneration through attenuating STAT3 phosphorylation in mice. Lab Invest 89, 1043-1052.

30 Ueki K, Kondo T, Tseng YH \& Kahn CR (2004) Central role of suppressors of cytokine signaling proteins in hepatic steatosis, insulin resistance, and the metabolic syndrome in the mouse. Proc Natl Acad Sci USA 10, 10422-10427. 
31 Torisu T, Sato N, Yoshiga D, Kobayashi T, Yoshioka T, Mori H, Iida M \& Yoshimura A (2007) The dual function of hepatic SOCS3 in insulin resistance in vivo. Genes Cells 12, 143-154.

32 Shi H, Cave B, Inouye K, Bjorbaek C \& Flier JS (2006) Overexpression of suppressor of cytokine signaling 3 in adipose tissue causes local but not systemic insulin resistance. Diabetes 55, 699-707.

33 Yang M, Zhang L, Wang C, Liu H, Boden G, Yang G \& Li L (2012) Liraglutide increases FGF-21 activity and insulin sensitivity in high fat diet and adiponectin knockdown induced insulin resistance. PLOS ONE 7, e48392.

34 Sun B, Yang G, Yang M, Liu H, Boden G \& Li L (2012) Long-term high-fat diet links the regulation of the insulin-sensitizing fibroblast growth factor-21 and visfatin. Cytokine 59, 131-137.

35 Muse ED, Lam TK, Scherer PE \& Rossetti L (2007) Hypothalamic resistin induces hepatic insulin resistance. $J$ Clin Invest 117, 1670-1678.

36 Ling L, Gangyi Y, Shaochuan S, Mengliu Y, Hua L \& Boden G (2009) The adipose triglyceride lipase, adiponectin and visfatin are downregulated by tumor necrosis factor- $\alpha$ (TNF- $\alpha)$ in vivo. Cytokine 45, 12-19.

37 Qin S, Ling L, Renzhe L, Mengliu Y, Hua L \& Gangyi Y (2009) Overexpression of visfatin/PBEF/Nampt alters whole-body insulin sensitivity and lipid profile in rats. Ann Med 41, 311-320.

38 Zhang L, Yang M, Ren H, Hu H, Boden G, Li L \& Yang G (2013) GLP-1 analogue prevents NAFLD in ApoE KO mice with diet and Acrp30 knockdown by inhibiting c-JNK. Liver Int 33, 794-804.

39 Ramesha CS, Paul R \& Ganguly J (1980) Effect of dietary unsaturated oils on the biosynthesis of cholesterol, and on biliary and fecal excretion of cholesterol and bile acids in rats. $J$ Nutr 110, 2149-2158.

40 Ferre T, Riu E, Bosch F \& Valera A (1996) Evidence from transgenic mice that glucokinase is rate limiting for glucose utilization in the liver. FASEB $J \mathbf{1 0}, 1213$ 1218.

\section{Supporting information}

Additional supporting information may be found in the online version of this article at the publisher's web site:

Fig. S1. Relative FGF-21 mRNA levels in the skeletal muscle of ApoE KO mice.

Fig. S2. Plasma glucose levels and glucose disposal rate during hyperinsulinemic/euglycemic clamping.

Table S1. Primers used for real-time PCR analysis. 